\title{
Las Comisiones de Monumentos y las Sociedades Arqueológicas como instrumentos para la construcción del pasado europeo*
}

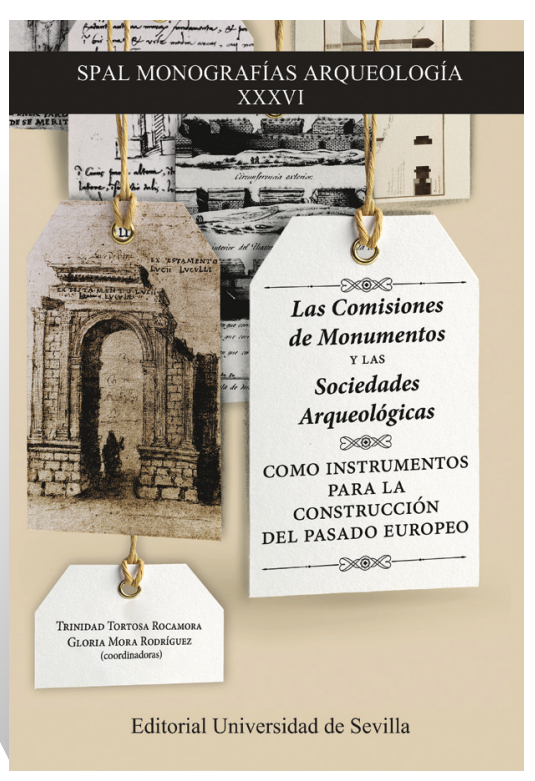

FICHA BIBLIOGRÁFICA

Tortosa Rocamora, Trinidad y Mora, Gloria (eds.), Las Comisiones de Monumentos y las Sociedades Arqueológicas como instrumentos para la construcción del pasado europeo, Sevilla, SPAL Monografías Arqueología, XXXVI, 2021, páginas 400, ISBN: 978-84-472-3024-2.

\section{Santiago Olcina Lagos I Universidad de Alicante}

El libro que a continuación reseñamos, publicado en 2021 y editado en el no XXXVI de la Colección SPAL Monografías Arqueología de la Universidad de Sevilla, recoge las aportaciones presentadas en la Reunión Científica Internacional que bajo el mismo título se celebró en Mérida los días 23 y 24 de noviembre de 2017, y organizaron las Dras. Trinidad Tortosa Rocamora

\footnotetext{
* Realizado en el marco de un proyecto de investigación con contrato FPU del Vicerrectorado de Investigación y Transferencia del Conocimiento de la Universidad de Alicante, y perteneciente al grupo de investigación "Arqueología y Patrimonio Histórico", durante una estancia de investigación en el Instituto de Arqueología de Mérida, CSIC-Junta de Extremadura, bajo la dirección de la Dra. Tortosa Rocamora.
} 
y Gloria Mora (Instituto de Arqueología de Mérida del CSIC-Junta de Extremadura y Universidad Autónoma de Madrid, respectivamente). El encuentro se planteó con la finalidad de revisar críticamente el papel que jugaron las Comisiones Provinciales de Monumentos de España y las Sociedades Arqueológicas en la formación del pasado nacional y europeo ${ }^{1}$, de actualizar el estado de la cuestión de las últimas tres décadas y, en consecuencia, de generar nuevos debates en torno al conocimiento y la importancia de estos agentes en la gestión institucional del patrimonio arqueológico. Un encuentro que supone, además, la continuación de otras obras y congresos de la misma categoría que cimentaron las bases literarias, metodológicas y científicas sobre los estudios de Historiografía Arqueológica en España, y cuyo desarrollo perfectamente han resumido sus coordinadoras en la introducción a este trabajo ${ }^{2}$.

La idea de esta publicación no es otra que la de contribuir al conocimiento mediante una nueva y actualizada perspectiva de esta línea de investigación. No obstante, debemos advertir al lector interesado que no estamos ante unas Actas al uso, aunque la finalidad primaria de este tipo de trabajos sí lo sea. Es decir, la de recoger en un documento único las aportaciones realizadas en el marco de estos congresos y servir, a la postre, como testimonio documental de su realización. En este caso no es así. Nos encontramos ante una publicación muy bien pensada y detallada en lo académico, en el que su hilo argumental liga y representa fielmente los objetivos propuestos para estas jornadas: servir de base metodológica para los futuros trabajos que se desarrollen sobre esta materia. Es una obra de referencia y consulta necesaria para conocer el actual punto de partida. Sin su obligada lectura no se comprenderían ni los planteamientos ni el marco por el que necesariamente está avanzando la investigación.

Y es que, el estudio de las Comisiones Provinciales de Monumentos de España y las Sociedades Arqueológicas precisaban de un nuevo enfoque que permitiera profundizar mucho

1. La revisión de estas cuestiones toma como base los resultados que aportaron las autoras en 1996 sobre la actuación de estos agentes en el territorio español (Trinidad Tortosa Rocamora y Gloria Mora, "La actuación de la Real Academia de la Historia sobre el patrimonio arqueológico: ruinas y antigüedades”, Archivo Español de Arqueología, 69, 1996, 191-217). Ellas se dieron cuenta, a partir de los indicadores bibliométricos, que este era uno de sus artículos más citados y de mayor impacto en la literatura científica, lo que ponía de manifiesto la actualidad de estos asuntos y afianzó indudablemente el propósito por el que se organizó el encuentro.

2. Véase, entre algunos otros ejemplos citados, Javier Arce Martínez y Ricardo Olmos Romera, Historiografía de la Arqueología y de la Historia Antigua en España (siglos XVIII-XX), Madrid, Instituto de Conservadores y Restauradores de Bienes Culturales, 1991; Fernando Gascó La Calle, José Beltrán Fortes y José Tomás Saracho Villalobos, La Antigüedad como argumento. Historiografía de Arqueología e Historia Antigua en Andalucía, Sevilla, Junta de Andalucía, 1993; José Beltrán Fortes y Fernando Gascó La Calle, La Antigüedad como argumento II. Historiografía de Arqueología e Historia Antigua en Andalucía, Cataluña, Scriptorium, 1995; Gloria Mora y Margarita Díaz-Andreu, La cristalización del pasado. Génesis y desarrollo del marco institucional de la arqueología en España, Málaga, Universidad de Málaga, 1997; José Beltrán Fortes, Beatrice Cacciotti, Xavier Dupré Raventós y Beatrice Palma Venetucci, Illuminismo e Ilustración. Le antichità e i loro protagonisti in Spagna e in Italia nel XVIII secolo, Roma, L’Erma di Bretschneider, 2003; José Beltrán Fortes, Beatrice Cacciotti y Beatrice Palma Venetucci, Arqueología, Coleccionismo y Antigüedad. España e Italia en el siglo XIX, Sevilla, Universidad de Sevilla, 2006. 
más en sus procesos de gestión, en su integración en el contexto nacional y en las posibles semejanzas y diferencias con sus organismos homólogos europeos. Ya han pasado más de treinta años desde que los estudios relacionados con la Historia y la Historiografía de la Arqueología se empezasen a publicar en nuestro país, y a día de hoy se cuenta con la madurez, el recorrido investigador y la bibliografía suficiente como para poder dar ese cambio y avanzar hacia nuevos escenarios. Atrás ha quedado el acercamiento a estas instituciones desde el punto de vista diacrónico y puramente provincial, centrado en aspectos intrínsecos a las respectivas comisiones y su relación exclusiva con su territorio. Los avances con los que cuenta a día de hoy la disciplina y la incorporación de nuevos instrumentos y herramientas para su conocimiento permiten abordar perfectamente estos trabajos desde nuevas perspectivas. Unas perspectivas en las que priman los enfoques dirigidos, como decimos, a sus procesos, sus protagonistas y su integración en el ámbito nacional y europeo. Las Comisiones Provinciales de Monumentos, las Sociedades Arqueológicas y el resto de instituciones destinadas a la gestión del patrimonio arqueológico tienen mucho más que una mirada, tienen mucho más que un recorrido. Estas comisiones son organizaciones poliédricas cuyo resultado final es el fruto de muchos y muy distintos factores, y de esta manera es como lo han querido transmitir y dejar patente todos los firmantes de la obra. La cual es, sin lugar a dudas, un reflejo de esta nueva metodología. Estos agentes implicados se tienen que entender, a partir de ahora, no como instituciones territoriales estancas que únicamente participan en su contexto más cercano y están ajenas a lo que ocurre fuera de él, sino como organismos abiertos, enmarcados en un contexto nacional y europeo del que participan y que inevitablemente configura su proceder interno (Fig. 1).

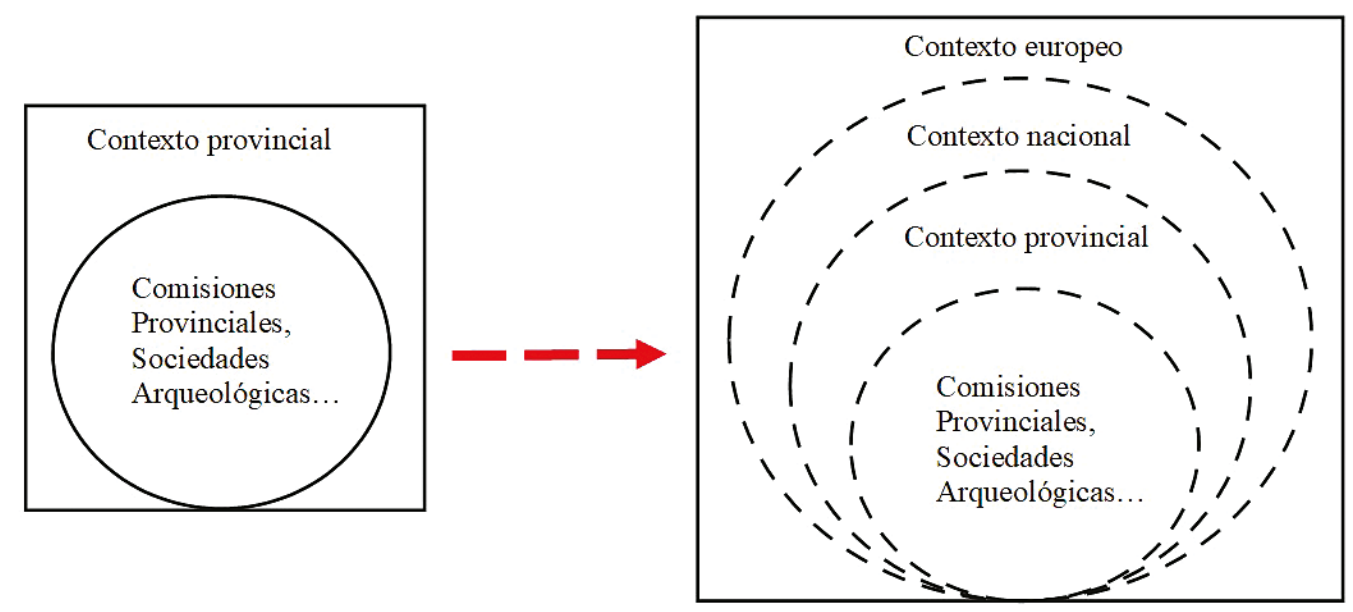

Fig. 1. Mapa conceptual referente al nuevo enfoque metodológico propuesto por Trinidad Tortosa Rocamora y Gloria Mora (2021). Elaboración propia a partir de los resultados de su trabajo. 
El libro que tenemos entre manos está estructurado en tres grandes apartados. Tres bloques temáticos en los que se organizó la reunión y con el que sus coordinadoras nos permiten ver, a simple vista, el esquema conceptual con el que desarrollaron el encuentro y al que líneas atrás hacíamos referencia.

Este se encuentra precedido por un interesante prólogo (pp. 9-12) realizado por Gonzalo Ruiz Zapatero (Universidad Complutense de Madrid-Sociedad Española de Historia de la Arqueología) en el que reflexiona sobre la Historiografía de la Arqueología y cómo sus estudios han contribuido al conocimiento de la configuración de nuestro pasado histórico; sobre la necesidad de repensar, gracias a estas nuevas miradas, el vínculo ininterrumpido entre anticuarismo y arqueología pre-científica o moderna, y sobre el papel que jugaron las Comisiones Provinciales de Monumentos de España en la construcción de todo este relato. Unas reflexiones que nos introducen directamente en el tema abordado en este libro y que alientan a continuar sobre estas líneas de investigación.

A él acompaña una introducción de sus autoras (pp. 13-16) en la que detallan las motivaciones que les llevaron a plantear el Congreso y en la que muestran los diferentes bloques temáticos en los que se articuló y en la que defienden la continuidad de estudios de este tipo. Una defensa muy acertada, tanto por plasmarse en una obra en la que precisamente se quiere mostrar la madurez y la actividad de la disciplina, como por la perspectiva de futuro que se le dibuja y los términos utilizados para ello. La Historiografía de la Arqueología debe dejar de verse, tal y como aclaran, como la simple historia de la investigación de un yacimiento y tiene que ser comprendida como una pieza imprescindible en los estudios histórico-arqueológicos. Estos nunca estarán completos si no están respaldados por un estudio historiográfico crítico en el que se profundice seriamente en los distintos y posibles procesos que intervienen en su desarrollo pasado, presente y futuro. Una serie de procesos que son los que realmente explican y dan sentido al yacimiento, al arqueólogo, a la colección o a la institución, entre sus muchos casos de estudio.

Por último, José María Luzón Nogué (Real Academia de Bellas Artes de San Fernando) cierra el libro con un epílogo (pp. 383-389) en el que sitúa a las Comisiones Provinciales de Monumentos en su contexto decimonónico nacional y europeo. Un final que nos permite integrar los trabajos plasmados en el libro desde la abstracción y su posición dentro del circuito de gestión del patrimonio arqueológico.

El primero de los capítulos (pp. 17-151), titulado «Identidades-Sociedades Arqueológicas-Comisiones de Monumentos», nos acerca al papel que desempeñó la Arqueología en la construcción de la identidad nacional y europea. A través de varios ejemplos podremos conocer cómo nuestra disciplina y sus elementos de gestión patrimonial se desarrollaron en España y en los países de su entorno como instrumentos para la construcción de sus discursos identitarios, pudiendo comparar los procesos y dejando entrever las posibles diferencias y semejanzas existentes entre ellos.

El primero de ellos es el de Carlos Fabiâo (Faculdade de Letras da Universidade de Lisboa) y en él hace un desarrollo del patrimonio arqueológico portugués a partir de la normativa de 1721, del concepto de Monumento Nacional y de los distintos tipos de vestigios que su administración consideraba necesario proteger bajo estas figuras jurídicas. 
Todo ello a través de algunas menciones específicas que nos permiten comprender el proceso que atravesó el país desde el siglo XVIII hasta las primeras décadas del XX.

Nathan Schlanger (École Nationale des Chartes) hace un recorrido por la Historia de la Arqueología y la Prehistoria francesa desde las primeras misiones de Napoleón I en Egipto hasta la segunda mitad del siglo XX; y muestra cómo estas disciplinas y sus impulsores fueron adaptando metodologías y herramientas de trabajo para su establecimiento, y para la comprensión de su identidad nacional. No sin sufrir, paralelamente, procesos de cambios legislativos que afectaron la forma de su proceder arqueológico.

Antonella Romani (Liceo Scientifico Statale "Nomentano" de Roma) explica el concepto italiano de «patrimonio nacional» en el siglo XIX a través de los impulsos de las administraciones encargadas de su gestión, como las Commissioni conservatrici dei Monumenti, las Soprintendenze o la Dirección de Antigüedades y Bellas Artes del Ministerio de Educación; así como de personajes que también contribuyeron en esta causa, como Giuseppe Fiorelli o Felice Barnabei.

Grégory Reimond (Casa Velázquez/Université de Toulouse-Jean Jaurès) hace un estudio sobre la participación de arqueólogos franceses en nuestro territorio desde finales del siglo XIX y la reformulación de este circuito que él propone en torno a la visión liberal del patrimonio arqueológico. Una visión que ya Gonzalo Ruiz Zapatero en el prólogo de esta obra ha querido destacar muy acertadamente por lo novedoso de su discurso y a la que le auguramos más de un debate en la comunidad científica.

Por su parte, Trinidad Tortosa y Gloria Mora también reflexionan sobre el papel de las Comisiones Provinciales de Monumentos de España y su participación en los procesos que configuraron la transformación de algunos monumentos en elementos fundamentales para la creación del discurso, difusión y consolidación del pasado histórico nacional, como pueden ser la Dama de Elche o la Gran Dama oferente del Cerro de los Santos. Dos espectaculares esculturas que a día de hoy sería imposible desvincular de nuestra identidad como pueblo y cuyo origen se encuentra, precisamente, en este proceso. Es por ello que es tan importante estudiar profundamente estas cuestiones y visibilizar el papel de la Historiografía, pues son ellas y no otras las que deben aportar luz en torno a la implicación de la Arqueología en el desarrollo identitario.

Como podemos comprobar, un interesantísimo compendio de estudios, que nos permiten calibrar y situar el tempo y el contexto de cada uno de estos países con la gestión de su patrimonio arqueológico y su participación dentro del circuito europeo.

El segundo bloque (pp. 153-301), titulado «Las Comisiones de Monumentos: particularidades generales y casos concretos», recoge aportaciones relacionadas con las Comisiones Provinciales de Monumentos y su implicación en los trabajos de gestión patrimonial. Especialmente, en lo que a la instalación y creación de los Museos Provinciales y excavaciones arqueológicas se refiere. A este respecto, tenemos que tener en cuenta que la materialización de estos espacios supuso para estas organizaciones el haber cumplido con uno de sus en- 
cargos más importantes ${ }^{3}$. Todas las obras procedentes de los conventos desamortizados, de hallazgos casuales y de excavaciones casuales o proyectadas por sus miembros debían reunirse en estos espacios para su conservación, estudio y difusión. Los Museos Provinciales y las excavaciones arqueológicas no se entienden, o no deberían entenderse, sin el constante y agotador estímulo de las Comisiones Provinciales de Monumentos. Estas actividades se configuran, por tanto, como uno de los elementos fundamentales para comprender su desarrollo en la gestión del patrimonio arqueológico en los siglos XIX y XX.

En relación a esto, podemos citar el interesante estudio de José Beltrán Fortes (Universidad de Sevilla) sobre la Diputación Arqueológica y la Comisión Provincial de Monumentos de Sevilla y su implicación con la Arqueología durante el siglo XIX y primeras décadas del XX, destacando ampliamente el caso de Itálica en sus particulares procesos de gestión.

También, el de Xavier Aquilué (Centre Iberia Graeca-Museu d’Arqueologia de Catalunya) sobre la Comisión Provincial de Monumentos de Gerona, la Junta de Museus de Barcelona y su relación con la gestión y recuperación de un enclave monumental tan importante como el de Empúries.

Pablo Ortiz Romero (Universidad de Extremadura) trata sobre la relación existente entre la política coyuntural del momento y el devenir de las Comisiones Provinciales de Monumentos. En este caso, de las comisiones extremeñas.

Blanca Gamo Parras (Museo de Albacete) explica los avatares por los que atravesó la Comisión Provincial de Monumentos de Albacete para poder reunir, conservar y exhibir las piezas y colecciones reunidas en su Museo.

Por último, José Antonio Fernández de Córdoba Pérez (Gobierno del Principado de Asturias) realiza una la novedosa comparación que realiza entre la Comisión Provincial de Monumentos de Asturias y el resto de sus homólogas españolas entre 1844 y 1918.

Todos ellos son asuntos que formaron parte de la historia de vida de estas instituciones y que han sido tratados desde una perspectiva diferente. Perspectiva que marca el camino por el que han de transitar los nuevos estudios referentes a las Comisiones Provinciales de Monumentos de España y el resto de organismos implicados en la gestión del patrimonio arqueológico.

El tercer bloque (pp. 303-381), titulado «Transmisión y comunicación social del patrimonio arqueológico», recoge dos aportaciones sobre la difusión del patrimonio arqueológico y sus inicios como activos turísticos.

Carlos J. Sánchez Morán (Instituto de Arqueología de Mérida, CSIC-Junta de Extremadura) a partir del caso de Mérida pone de relieve el papel que tuvo la Subcomisión Provincial de Monumentos en el proyecto de excavación y difusión arqueológica desarrollado en la ciudad a partir de 1910; y cómo gracias a otro tipo de documentación que no es la que

3. Según las Reales Órdenes por las que se ordenaron las Comisiones Provinciales: las del 13 de junio y 24 de julio de 1844 (Gaceta de Madrid, núm. 3568 de 21 de junio de 1844; núm. 3605 de 28 de julio de 1844). Así como sus sucesivas reorganizaciones del 24 de noviembre de 1865 (Gaceta de Madrid, núm. 345 de 11 de diciembre de 1865), del 11 de agosto de 1918 (Gaceta de Madrid, núm. 226 de 14 de agosto de 1918) y del 26 de marzo de 1929 (Gaceta de Madrid, núm. 94 de 4 de abril de 1929), respectivamente. 
oficialmente generó esta Subcomisión se han podido reconstruir algunos interesantísimos episodios de su historia de vida. Un discurso que ha permitido rebatir las ideas que hasta entonces se habían planteado para esta Subcomisión, y ofrecer una visión renovada y acorde a su verdadero conocimiento historiográfico.

Por su parte, Ignacio Rodríguez Temiño, Juan Antonio Pachón Romero y José Ildefonso Ruiz Cecilia (Conjunto Arqueológico de Carmona) analizan el interés de las élites burguesas en la conservación y la recuperación del patrimonio arqueológico a través de los restos y los hallazgos encontrados en Carmona y Osuna a finales del siglo XIX. Un interés que en muchas ocasiones fue el estímulo para la gestión de estos monumentos, especialmente en esta centuria, y cuyo resultado para estos casos sería muy interesante poder contrastarlo en un futuro no muy lejano con el resto de comisiones españolas.

En resumen, nos encontramos ante una publicación que actualiza el conocimiento historiográfico en relación al patrimonio arqueológico y los agentes implicados en su gestión en los siglos XIX y XX (Comisiones Provinciales, Diputaciones, Sociedades Arqueológicas, Academias, personajes, etc.). Una publicación que marca un punto de inflexión en la metodología de estudio de estas cuestiones, y que abre nuevas vías, interrogantes y perspectivas de futuro muy prometedoras para la Historiografía Arqueológica. Esperamos que esta nueva línea de trabajo tenga una gran acogida, sea de interés para la comunidad investigadora y nos permita seguir acrecentando el conocimiento de esta disciplina, pudiéndola situar al frente en este tipo de estudios. 